\title{
Ehrbarer Staat? Eine Zwischenbilanz schwarz-gelber Regierungspolitik
}

\author{
von Stefan Moog und Bernd Raffelhüschen
}

\begin{abstract}
Nach einem Drittel der Legislaturperiode scheint die im Herbst 2009 angetretene schwarz-gelbe Koalition aus CDU, CSU und FDP mit dem „Wachstumsbeschleunigungsgesetz“, dem „Zukunftspaket“ und der Gesundheitsreform wesentliche Ziele des Koalitionsvertrages realisiert zu haben. Gleichwohl fällt die fiskalische Bilanz von SchwarzGelb bislang deutlich ernüchternd aus. Bei einem vernachlässigbaren Einfluss auf die Wirtschaftsleistung bürdete das „Wachstumsbeschleunigungsgesetz“" den öffentlichen Haushalten zusätzliche Lasten von jährlich $8 \mathrm{Mrd}$. Euro auf. Insofern ist es zu begrüßen, dass die Regierungskoalition mit dem „Zukunftspaket“ eine Strategie zur Konsolidierung der öffentlichen Haushalte umzusetzen sucht. Allerdings haben die bisher verwirklichten Bausteine dieser Konsolidierungsstrategie zu einer nur geringen Entlastung der öffentlichen Hand geführt. Wirklichen Erfolg wird man der Bundesregierung erst dann zusprechen können, wenn es ihr gelingt, die geplanten, bisher nur als „Luftbuchungen“ zu kennzeichnenden Konsolidierungsmaßnahmen in die Tat umzusetzen. Als unzureichend erweist sich zudem die Gesundheitsreform. Durch Beitragssatzerhöhungen und kurzfristige Kostendämpfungsmaßnahmen werden im Wesentlichen erneut nur die Symptome der strukturellen Mängel des Gesundheitssystems behandelt.
\end{abstract}

During the first 16 months of its term in office, the governing coalition of CDU, CSU and FDP has addressed three important goals mentioned in the October 2009 coalition agreement: it has implemented a bill providing for an acceleration of economic growth, designed an agenda of fiscal retrenchment aiming at meeting future economic challenges, and passed a health care reform bill. However, the fiscal record of these reforms remains bleak. While its effect on economic performance was negligible, the "Wachstumsbeschleunigungsgesetz" caused an additional annual gap of 8 bn. euro in the federal budget. The "Zukunftspaket" was to address this issue by proposing a strategy for fiscal consolidation. Nonetheless, it has failed to cause any major effects thus far. In addition, the health care reform failed to address the underlying problems of the medical system and merely treated a few immediate symptoms. Therefore, much room for improvement remains and the government will need to make use of the given opportunities if it seeks to implement its objectives.

Anmerkung der Autoren: Für wertvolle Hinweise und Hilfestellungen danken wir Tobias Benz, Arne Leifels, Tobias Hackmann, Christian Hagist, Susanna Hübner, Christoph Müller, Guido Raddatz, Jörg Schoder und Johannes Vatter. 


\section{Einleitung}

Rückblickend hätte die wirtschaftliche und fiskalische Ausgangslage für die christlich-liberale Koalition bei ihrem Amtsantritt nicht ungünstiger ausfallen können. Mit einem Rückgang der realen Wirtschaftsleistung um fast fünf Prozent im Jahr 2009 steckte die deutsche Volkswirtschaft zum damaligen Zeitpunkt inmitten der schwersten Rezession der Nachkriegszeit. Und obwohl die Wirtschaftsprognosen für das Jahr 2010 zumindest Anlass für verhaltenen Optimismus boten, lastete auf der neuen Bundesregierung infolge der noch von der Großen Koalition aus CDU, CSU und SPD beschlossenen Konjunkturprogramme bereits die Bürde einer erwarteten Rekordneuverschuldung von $86 \mathrm{Mrd}$. Euro. An sich hätte dies Ansporn genug sein müssen, um auf der Grundlage mutiger Entscheidungen die durch die Schuldenbremse auferlegten Vorgaben für die Haushaltskonsolidierung sowie die dringend notwendigen Reformen der Kranken- und Pflegeversicherung anzugehen.

Doch weit gefehlt: Mit dem „Wachstumsbeschleunigungsgesetz” bürdete die neu gewählte Regierungskoalition den Haushalten der Gebietskörperschaften zusätzliche Lasten von jährlich $8 \mathrm{Mrd}$. Euro auf. Angefangen beim Streit um die im Koalitionsvertrag vereinbarte Steuerentlastung bis hin zur Reformbaustelle Gesundheitspolitik zeigte sich, wie weit die Positionen der Koalitionspartner tatsächlich auseinander lagen, während man bei der Frage der Haushaltskonsolidierung lediglich auf Zeit spielte, um die Gunst der Bürger bzw. Wähler angesichts der im Mai 2010 bevorstehenden wichtigen Landtagswahl in NordrheinWestfalen nicht zu verspielen. Diese Rechnung ging jedoch nicht auf. Fiskalpolitische Konzeptlosigkeit und interner Streit prägten das öffentliche Erscheinungsbild bereits in den ersten Monaten. Die Konsolidierung der Staatsfinanzen und die Reform der Sozialversicherungen blieben dabei größtenteils auf der Strecke.

Vor diesem Hintergrund zieht der vorliegende Beitrag auf Grundlage der Methodik der Generationenbilanzierung ein fiskalisches Zwischenfazit der bisherigen Arbeit von Schwarz-Gelb. ${ }^{1}$ In Abschnitt II wird als Bestandsaufnahme zunächst die fiskalische Ausgangslage zu Beginn der Legislaturperiode dargestellt. Die fiskalischen Wirkungen der drei zentralen wirtschaftspolitischen Gesetzesvorhaben der Bundesregierung werden in Abschnitt III untersucht. Neben dem

1 Eine Beschreibung der Methodik wie auch der Kritik an der Generationenbilanzierung findet sich bei Raffelhüschen, B.: Generational Accounting: Method, Data and Limitations, in: European Economy, Reports and Studies, 6 (1999), 17-28. 
„Wachstumsbeschleunigungsgesetz” zählen hierzu das als „Zukunftspaket“ bezeichnete Konsolidierungsprogramm sowie die Gesundheitsreform. Die Studie schließt in Abschnitt IV mit einem Fazit sowie einem Ausblick auf (weiterhin) dringend gebotene Schritte zur Bewältigung der Konsolidierungsaufgabe.

\section{Das fiskalische Erbe der Großen Koalition}

Mit ihren Renten- und Arbeitsmarktreformen hatte die rot-grüne Bundesregierung (1998- 2005) unter Bundeskanzler Gerhard Schröder die Weichen für eine nachhaltige Finanzierung der Gesetzlichen Rentenversicherung (GRV) gestellt und das Fundament für das aktuelle „Jobwunder“ auf dem Arbeitsmarkt gelegt. Im Vergleich dazu nimmt sich die Reformbilanz der Großen Koalition (20052009) eher bescheiden aus. Dennoch wurde mit der „Rente mit 67“ der durch Rot-Grün eingeleitete Rentenreformprozess immerhin fortgeführt und mit der Unternehmensteuerreform 2008 eine Verbesserung des damaligen gesetzlichen status quo erzielt. Schließlich gab es auch Erfolge bei der Konsolidierung der öffentlichen Haushalte. So konnte die Zunahme der Staatsausgaben während der Amtszeit der Großen Koalition gebremst und die Staatsquote von 46,8 Prozent des BIP im Jahr 2005 auf 43,8 Prozent des BIP im Jahr 2008 gesenkt sowie das Defizit des öffentlichen Gesamthaushalts in den Jahren 2007 und 2008 erstmals seit 1989 wieder auf eine ,rote“ Null zurückgeführt werden. ${ }^{2}$

Zwar waren die Konsolidierungserfolge von Schwarz-Rot in Teilen auch der guten konjunkturellen Entwicklung und der Erhöhung des Mehrwertsteuersatzes von 16 auf 19 Prozent in 2007 geschuldet, doch sind sie nicht ausschließlich als kurzfristige Effekte abzutun. Wie Abbildung 1 zeigt, konnte auch die Divergenz zwischen der langfristigen Entwicklung der Einnahmen und Ausgaben der öffentlichen Haushalte in den ersten drei Amtsjahren der Großen Koalition erkennbar reduziert werden. Belief sich die Nachhaltigkeitslücke zu Beginn der Amtszeit von Schwarz-Rot noch auf 350,1 Prozent des BIP (Basisjahr 2004), so sank sie binnen zwei Jahren auf einen Tiefstand von 188,8 Prozent (Basis: 2006). ${ }^{3}$ Infolge der Konjunkturprogramme zur Bewältigung der Wirtschafts- und Finanzkrise trübte sich die erfreuliche Bilanz der Großen Koalition in ihrem letzten

2 Zwar wies der öffentliche Gesamthaushalt zuletzt im Jahr 2000 einen Überschuss von etwa 1,3 Prozent des BIP auf, doch ist dieser auf die einmaligen Einnahmen aus dem Verkauf der UMTS-Lizenzen zurückzuführen.

3 Für eine detaillierte Beschreibung der zugrundegelegten Annahmen und Nachhaltigkeitsindikatoren s. Stiftung Marktwirtschaft: Ehrbarer Staat? Die Generationenbilanz - Update 2010: Handlungsoptionen der Gesundheitspolitik, in: Argumente zu Marktwirtschaft und Politik, Nr. 111 (2010). 
Amtsjahr allerdings deutlich ein, die Nachhaltigkeitslücke nahm bis zum Ende der Legislaturperiode wieder auf 291 Prozent zu (Basis: 2008).

Abbildung 1: Das fiskalische Erbe der Großen Koalition

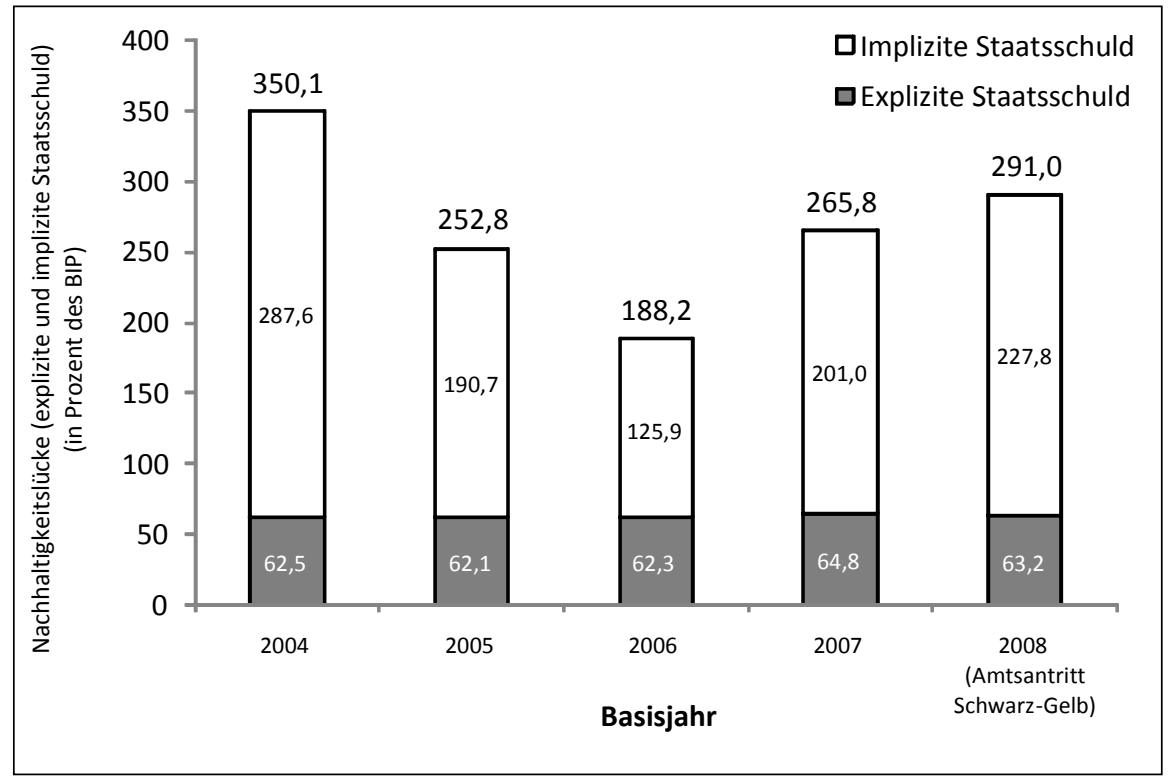

Anmerkung: $\mathrm{g}=1,5 \%, \mathrm{r}=3,0 \%, 12$. koordinierte Bevölkerungsvorausberechnung des Statistischen Bundesamtes. Quelle: Eigene Berechnungen.

\section{Eine erste Bilanz der schwarz-gelben Koalition}

Am 26. Oktober 2009 unterzeichneten die Vorsitzenden von CDU, CSU und FDP den nach der Bundestagswahl ausgehandelten Koalitionsvertrag. Gemessen an den dort formulierten Zielen kann man der christlich-liberalen Koalition bisher zumindest keine Untätigkeit vorwerfen. Mit dem „Wachstumsbeschleunigungsgesetz" hat die Bundesregierung wesentliche Elemente des dort vereinbarten Sofortprogramms zur Bekämpfung der Finanz- und Wirtschaftskrise zeitnah umgesetzt und mit dem „Zukunftspaket“ ein Konsolidierungsprogramm verabschiedet, das zumindest auf dem Papier den Anforderungen der Schuldenbremse genügt. Schließlich wurde mit der Gesundheitsreform auch die im Koalitionsvertrag vereinbarte Reform der Gesetzlichen Krankenversicherung angegangen. Im Folgenden werden diese drei zentralen Maßnahmenpakete aus der Anfangsphase der schwarz-gelben Regierung jeweils kurz dargestellt, bewertet und auf ihre Konsequenzen für die Nachhaltigkeit der öffentlichen Haushalte untersucht. 


\section{Das „Wachstumsbeschleunigungsgesetz“ oder die Bilanz der ersten Beschlüsse}

Angesichts der „Folgen der schwersten Wirtschafts- und Finanzkrise“ verfolgte die Bundesregierung mit dem „Wachstumsbeschleunigungsgesetz“ (WaBeG) das Ziel, „,neue Impulse für einen stabilen und dynamischen Aufschwung zu setzen“, indem „durch wirksame und zielgerichtete steuerliche Entlastungen die produktiven Kräfte unserer Gesellschaft" gestärkt werden. ${ }^{4} \mathrm{Zu}$ diesem Zweck wurde mit dem $\mathrm{WaBeG}$ zum einen das im Koalitionsvertrag angekündigte Sofortprogramm zur Bekämpfung der Finanz- und Wirtschaftskrise umgesetzt, mit dem verschiedene Wachstumshemmnisse im Bereich der Unternehmensbesteuerung beseitigt werden sollten. Zum anderen wurde mit dem $\mathrm{WaBeG}$ auch der erste Schritt für das im Koalitionsvertrag vereinbarte Ziel einer Steuererleichterung für Familien und Bezieher unterer und mittlerer Einkommen beschlossen.

Tabelle 1: Fiskalische Wirkung des „Wachstumsbeschleunigungsgesetzes“ (in Mrd. Euro)

\begin{tabular}{l|l|c|c|c|c}
\hline \multicolumn{2}{|c|}{} & 2011 & 2012 & 2013 & 2014 \\
\hline $\mathbf{A )}$ & Private Haushalte & $\mathbf{4 , 9}$ & $\mathbf{5 , 1}$ & $\mathbf{5 , 1}$ & $\mathbf{5 . 1}$ \\
\hline & Kindergeld/-freibeträge & 4,5 & 4,7 & 4,7 & 4,7 \\
\hline & $\begin{array}{l}\text { Geänderte Erbschaftsteuersätze für } \\
\text { Geschwister und Geschwisterkinder }\end{array}$ & 0,4 & 0,4 & 0,4 & 0,4 \\
\hline B) & Unternehmen & $\mathbf{3 , 3}$ & $\mathbf{3 , 9}$ & $\mathbf{3 , 3}$ & $\mathbf{3 , 1}$ \\
\hline & $\begin{array}{l}\text { Sanierungsklausel, Konzernklausel und } \\
\text { "Stille Reserven Escape" }\end{array}$ & 1,0 & 1,5 & 1,5 & 1,5 \\
\hline & $\begin{array}{l}\text { Ermäßigter Umsatzsteuersatz für Beherbergungsleistun- } \\
\text { gen }\end{array}$ & 1,0 & 1,0 & 1,0 & 1,0 \\
\hline & $\begin{array}{l}\text { Verbesserte Möglichkeiten zur Abschreibung gering- } \\
\text { wertiger Wirtschaftsgüter }\end{array}$ & 0,8 & 0,7 & 0,2 & 0,0 \\
\hline & Änderungen bei der Zinsschranke & 0,3 & 0,4 & 0,4 & 0,4 \\
\hline & $\begin{array}{l}\text { Reduktion des Hinzurechnungssatzes bei Immobilien- } \\
\text { mieten }\end{array}$ & 0,1 & 0,1 & 0,1 & 0,1 \\
\hline & Sonstige (Biokraftförderung, ...) & 0,1 & 0,2 & 0,1 & 0,1 \\
\hline Gesamtbelastung von Bund, Ländern und Gemeinden & $\mathbf{8 . 2}$ & $\mathbf{9 , 0}$ & $\mathbf{8 , 3}$ & $\mathbf{8 , 2}$ \\
\hline
\end{tabular}

Quelle: BT-Drucks. 17/15, eigene Berechnungen.

In der Würdigung umfasst das $\mathrm{WaBeG}$ verschiedene Einzelmaßnahmen, auf deren Grundlage die privaten Haushalte und Unternehmen im Umfang von jährlich $8 \mathrm{Mrd}$. Euro entlastet werden (siehe Tabelle 1). Die privaten Haushalte profitieren im Wesentlichen von der Anhebung des Kindergeldes um 20 Euro 
pro Kind und der Erhöhung der Kinderfreibeträge von 6.024 auf 7.008 Euro. Allein auf diese Maßnahme entfällt mit 4,7 Mrd. Euro mehr als die Hälfte der gesamten Entlastungswirkung des WaBeG. Zusätzlich schafft die Absenkung der Erbschaftsteuersätze für Geschwister und deren Kinder Entlastungen von jährlich etwa 400 Mio. Euro.

Aus Sicht der Unternehmen wurden mit dem $\mathrm{WaBeG}$ vor allem die bereits von der Großen Koalition als Teil des „Bürgerentlastungsgesetzes“ beschlossenen, aber befristeten Ausnahmeregelungen von der Unternehmensteuerreform 2008 dauerhaft umgesetzt. Hierunter fallen etwa die verbesserten Verlustabzugsbeschränkungen bei der Übertragung von Beteiligungsrechten und Lockerungen bei der Zinsschranke, mit denen die Unternehmen im Umfang von jährlich 1,9 Mrd. Euro entlastet werden. Auch kommt es zu einer weiteren Erleichterung durch die Anwendung des ermäßigten Umsatzsteuersatzes auf Beherbergungsdienstleistungen um jährlich 1 Mrd. Euro.

Insgesamt stellen die mit dem $\mathrm{WaBeG}$ beschlossenen Änderungen an der Unternehmensteuerreform einen Schritt in die richtige Richtung dar. ${ }^{5}$ Die Bevorzugung der Hoteliers durch die Herabsetzung des Umsatzsteuersatzes auf Beherbergungsdienstleistungen muss hingegen als Bedienung eines Partikularinteresses betrachtet werden, das sich ökonomisch nicht rechtfertigen lässt. Diese Maßnahme ist umso unverständlicher, als der Koalitionsvertrag als ein Ziel der Legislaturperiode eine Reform der Umsatzsteuer vorsieht, im Zuge derer alle Ausnahmen mit ermäßigtem Umsatzsteuersatz auf den Prüfstand gestellt werden sollen. Schließlich muss auch die mit der Anhebung des Kindergeldes und der Kinderfreibeträge beschlossene steuerliche Entlastung der privaten Haushalte hinterfragt werden. Da allein diese Maßnahme die öffentlichen Haushalte im Umfang von knapp 5 Mrd. Euro jährlich belastet, hätte man angesichts der notwendigen Haushaltskonsolidierung auf diese Steuererleichterung besser verzichtet. Dies gilt umso mehr, als die privaten Haushalte bereits durch die von der Großen Koalition als Teil des Konjunkturpakets II beschlossenen Steuersenkungen, die Wiedereinführung der Entfernungspauschale und die volle Absetzbarkeit der Kranken- und Pflegeversicherungsbeiträge beginnend mit dem Jahr 2010 um jährlich etwa $20 \mathrm{Mrd}$. Euro entlastet werden. Darüber hinaus muss die Frage

5 Vgl. hierzu Sachverständigenrat zur Begutachtung der gesamtwirtschaftlichen Entwicklung: Chancen für einen stabilen Aufschwung - Jahresgutachten 2010/11, Wiesbaden, 2010, sowie Kessler, W./Lindemer, J.: Die Zinsschranke nach dem Wachstumsbeschleunigungsgesetz, in: Der Betrieb, 9 (2010), 472-476. 
gestellt werden, ob dieses Geld im Sinne einer effizienten Familienförderung nicht zielgenauer für andere familienpolitische Maßnahmen wie etwa den Ausbau der Kinderbetreuung hätte eingesetzt werden können. ${ }^{6}$

Im Fazit hat das „Wachstumsbeschleunigungsgesetz“ seinen Namen nicht verdient. So stellen sachkundige Beobachter fest, dass das $\mathrm{WaBeG}$,nur einen verschwindend geringen Effekt auf das Bruttoinlandsprodukt“ hat und lediglich „,zu einer starken Ausweitung des strukturellen Defizits“ führt. ${ }^{7}$ Diese Einschätzung spiegelt sich auch deutlich in Abbildung 2 (s.u.) wider. Denn unabhängig von der Beurteilung der einzelnen Maßnahmen hat Schwarz-Gelb den öffentlichen Haushalten mit dem WaBeG vor allem eine zusätzliche Hypothek aufgebürdet, die sich in einer Zunahme der Nachhaltigkeitslücke von 291 Prozent des BIP bei Amtsantritt von Schwarz-Gelb auf nun 315 Prozent niederschlägt. Politisch mag es verständlich sein, dass die christlich-liberale Koalition den im Wahlkampf formulierten Versprechen auf eine steuerliche Entlastung auch Taten folgen lassen wollte. Allerdings war angesichts der Faktenlage von vornherein klar, dass für die Umsetzung dieser Steuerentlastungsversprechen kaum Spielraum existierte. Steuerentlastungen wären nur dann umsetzbar gewesen, wenn diese Mittel im Gegenzug an anderer Stelle eingespart worden wären.

\section{Das „Zukunftspaket“ - alles nur „heiße“ Luft?}

Vor diesem Hintergrund war es begrüßenswert, dass die schwarz-gelbe Regierungskoalition sich im Juni 2010 angesichts der fallenden Umfragewerte eines Besseren besonnen und mit dem in Eckpunkten formulierten Konsolidierungsprogramm zumindest bei den expliziten Staatsschulden eine Änderung in ihrer Prioritätensetzung eingeleitet hat. Angesichts der Vorgaben der Schuldenbremse war diese Kehrtwende auch verfassungsrechtlich geboten.

Im Kern sieht die Schuldenbremse vor, dass das sogenannte strukturelle - also um konjunkturelle Einflüsse bereinigte - Defizit des Bundes ab dem Jahr 2016 einen Betrag von 0,35 Prozent des BIP nicht überschreiten darf. ${ }^{8}$ Diesen Anfor-

6 So bemängelte etwa die OECD, dass die deutsche Familienpolitik bereits heute im Wesentlichen auf Geld- statt auf Sachleistungen setzt. Vgl. unter OECD: Babies and Bosses - Reconciling Work and Family Life, Paris, 2007.

7 Vgl. Hübner, M.: Das Wachstumsbeschleunigungsgesetz: Eine makroökonometrische Analyse, in: Wirtschaftsdienst, 90 (2010), 240-247.

8 Für die Bundesländer fordert die neue Schuldenregel ab dem Jahr 2020 einen strukturell ausgeglichenen Haushalt. 
derungen der neuen Schuldenregel wurde die Bundesregierung mit ihrem als „Zukunftspaket“ bezeichneten Konsolidierungsprogramm zumindest auf dem Papier gerecht. Insgesamt umfasst das Paket unterschiedliche Maßnahmen, durch die der Bundeshaushalt im Zeitraum 2011 bis 2014 bereits im Umfang von 80 Mrd. Euro entlastet werden soll.

Als einen ersten Schritt zur Umsetzung des „Zukunftspakets“ beschloss die Bundesregierung mit ihrer Mehrheit im Bundestag im Dezember 2010 das Haushaltsbegleitgesetz (HBeglG) und das Kernbrennstoffsteuergesetz (KernBStG).

Tabelle 2: Das „Zukunftspaket“ der Bundesregierung (in Mrd. Euro)

\begin{tabular}{|c|c|c|c|c|}
\hline in Mrd. Euro & 2011 & 2012 & 2013 & 2014 \\
\hline Subventionsabbau und ökologische Neujustierung & 2,3 & 2,2 & 2,5 & 2,5 \\
\hline Energie- und Stromsteuer & 1,3 & 1,2 & 1,5 & 1,5 \\
\hline Luftverkehrsteuer & 1,0 & 1,0 & 1,0 & 1,0 \\
\hline Beteiligung von Unternehmen & 3,1 & 5,2 & 5,2 & 5,2 \\
\hline Kernbrennstoffsteuer & 2,3 & 2,3 & 2,3 & 2,3 \\
\hline Finanzmarkttransaktionssteuer & 0,0 & 2,0 & 2,0 & 2,0 \\
\hline Bahndividende & 0,5 & 0,5 & 0,5 & 0,5 \\
\hline Fiskusprivileg im Insolvenzverfahren & 0,3 & 0,4 & 0,4 & 0,4 \\
\hline Neujustierung von Sozialgesetzen & 3,1 & 7,2 & 9,6 & 10,3 \\
\hline Ersatz von Pflicht- durch Ermessensleistungen & 2,0 & 4,0 & 5,0 & 5,0 \\
\hline $\begin{array}{l}\text { Effizienzverbesserungen bei der Arbeitsmarktvermitt- } \\
\text { lung }\end{array}$ & 0,0 & 0,0 & 1,5 & 3,0 \\
\hline Wegfall des Zuschlags beim Übergang ALG1 $\rightarrow$ ALG2 & 0,2 & 0,2 & 0,2 & 0,2 \\
\hline Wegfall Rentenversicherungspflicht ALG2 & 1,9 & 1,8 & 1,8 & 1,8 \\
\hline $\begin{array}{l}\text { Elterngeld (Absenkung Ersatzquote; Nicht- } \\
\text { Berücksichtigung bestimmter Einnahmen) }\end{array}$ & 0,2 & 0,2 & 0,2 & 0,2 \\
\hline $\begin{array}{l}\text { Anrechnung des Elterngeldes auf Leistungen zur Siche- } \\
\text { rung des Lebensunterhalts }\end{array}$ & 0,5 & 0,5 & 0,5 & 0,5 \\
\hline Wohngeld (Wegfall der Heizkostenkomponente) & 0,1 & 0,3 & 0,3 & 0,3 \\
\hline $\begin{array}{l}\text { Wegfall der Erstattung der Aufwendungen für eini- } \\
\text { gungsbedingte Leistungen }\end{array}$ & 0,3 & 0,3 & 0,2 & 0,2 \\
\hline Rückwirkung auf RV-Bundeszuschüsse & $-0,1$ & $-0,1$ & 0,0 & $-0,8$ \\
\hline Weitere Beteiligung des Bundes an den Kosten d. GKV & $-2,0$ & 0,0 & 0,0 & 0,0 \\
\hline Streitkräftereform & $\mathbf{0 , 0}$ & $\mathbf{0 , 0}$ & $\mathbf{1 , 0}$ & $\mathbf{3 , 0}$ \\
\hline Einsparungen im Verwaltungsbereich & 2,3 & 3,3 & 3,9 & 3,9 \\
\hline Einsparung bei disponiblen Ausgaben & 1,5 & 2,5 & 3,1 & 3,1 \\
\hline Verwaltungsausgaben & 0,8 & 0,8 & 0,8 & 0,8 \\
\hline Weitere Maßnahmen & $\mathbf{0 , 6}$ & 1,1 & 1,7 & 2,0 \\
\hline Zinsausgaben & 0,5 & 1,0 & 1,5 & 2,0 \\
\hline Stadtschloss & 0,1 & 0,1 & 0,2 & 0,0 \\
\hline Gesamtentlastung von Bund, Ländern und Gemeinden & $\mathbf{1 1 , 5}$ & 18,9 & 23,9 & 26,9 \\
\hline
\end{tabular}

Quelle: Bundesministerium für Finanzen. 
Mit dem KernBStG wurde befristet bis zum Jahr 2016 eine neue Steuer auf Kernbrennelemente - die sogenannte Kernbrennstoffsteuer - eingeführt, mit der dem Bundeshaushalt zusätzliche Einnahmen von jährlich bis zu 2,3 Mrd. Euro zufließen sollen. ${ }^{9}$ Durch die Befristung hat die Kernbrennstoffsteuer jedoch nur eine temporäre Entlastung des Bundeshaushalts zur Folge. Das HBeglG hingegen setzt sich aus den in Tabelle 3 dargestellten Einzelmaßnahmen zusammen. Diese können grob in einnahmeseitige und ausgabenseitige Maßnahmen unterteilt werden.

Tabelle 3: Erster Schritt des „Zukunftspakets“: Das Haushaltsbegleitgesetz

\begin{tabular}{|c|c|c|c|c|c|}
\hline \multicolumn{2}{|c|}{ in Mrd. Euro } & 2011 & 2012 & 2013 & 2014 \\
\hline & Einnahmeseitige Maßnahmen & 2,7 & 2,6 & 2,9 & 2,9 \\
\hline & Luftverkehrsteuer & 1,0 & 1,0 & 1,0 & 1,0 \\
\hline & Energie- und Stromsteuer & 1,3 & 1,2 & 1,5 & 1,5 \\
\hline & Fiskusprivileg im Insolvenzverfahren & 0,3 & 0,4 & 0,4 & 0,4 \\
\hline B) & Ausgabenseitige Maßnahmen & 1,0 & 1,1 & 1,1 & 1,1 \\
\hline & $\begin{array}{l}\text { Elterngeld (Absenkung Ersatzquote; Nicht- } \\
\text { Berücksichtigung bestimmter Einnahmen) }\end{array}$ & 0,2 & 0,2 & 0,2 & 0,2 \\
\hline & $\begin{array}{l}\text { Anrechnung des Elterngeldes auf Leistungen zur } \\
\text { Sicherung des Lebensunterhalts }\end{array}$ & 0,5 & 0,5 & 0,5 & 0,5 \\
\hline & $\begin{array}{l}\text { Wegfall des befristeten Zuschlags beim Übergang } \\
\text { von ALG1 zu ALG2 }\end{array}$ & 0,2 & 0,2 & 0,2 & 0,2 \\
\hline & Wegfall der Heizkostenkomponente beim Wohngeld & 0,1 & 0,3 & 0,3 & 0,3 \\
\hline C) & $\begin{array}{l}\text { Verschiebebahnhöfe } \\
\text { (Entlastung des Bundes zu Lasten der Sozialversi- } \\
\text { cherungen) }\end{array}$ & $\mathbf{0 , 1}$ & 2,1 & 2,0 & 1,2 \\
\hline & $\begin{array}{l}\text { Weitere Beteiligung des Bundes an den Kosten der } \\
\text { GKV für das Jahr } 2011\end{array}$ & $-2,0$ & 0,0 & 0,0 & 0,0 \\
\hline & Wegfall der Rentenversicherungspflicht bei ALG2 & 1,9 & 1,8 & 1,8 & 1,8 \\
\hline & $\begin{array}{l}\text { Wegfall der Erstattung der Aufwendungen für eini- } \\
\text { gungsbedingte Leistungen }\end{array}$ & 0,3 & 0,3 & 0,2 & 0,2 \\
\hline & Rückwirkung auf die Bundeszuschüsse an die GRV & $-0,1$ & $-0,1$ & 0,0 & $-0,8$ \\
\hline $\begin{array}{l}\text { Ges } \\
\text { von }\end{array}$ & $\begin{array}{l}\text { amte Entlastung } \\
\text { Bund, Ländern und Gemeinden }\end{array}$ & 3.8 & 5,7 & 6,0 & 5,2 \\
\hline & $\begin{array}{l}\text { ektive Entlastung } \\
\text { öffentlichen Gesamthaushalts }\end{array}$ & 3.7 & 3,7 & 4,0 & 4,0 \\
\hline
\end{tabular}

Quelle: BT-Drucks. 17/3030, eigene Berechnungen.

9 Durch die im März 2011 beschlossene Stilllegung der ältesten Atommeiler und das LaufzeitMoratorium dürfte der Verbrauch an Kernbrennelementen und damit das Aufkommen der Kernbrennstoffsteuer zumindest mittelfristig geringer ausfallen als von der Bundesregierung bei Verabschiedung des KernBStG veranschlagt. 
$\mathrm{Zu}$ den einnahmeseitigen Maßnahmen zählen die Einführung der Luftverkehrsteuer, das Streichen verschiedener Ausnahmetatbestände bei der Energie- und Stromsteuer sowie die Wiedereinführung des Fiskusprivilegs im Insolvenzverfahren. Hieraus ergibt sich insgesamt eine Entlastung der öffentlichen Haushalte i.H.v. 2,9 Mrd. Euro. Demgegenüber fallen die Kürzungen auf der Ausgabenseite mit insgesamt 1,1 Mrd. Euro vergleichsweise gering aus. Im Einzelnen fallen hierunter die Anrechnung des Elterngeldes auf Leistungen zur Sicherung des Lebensunterhalts, die Absenkung der Ersatzquote beim Elterngeld, der Wegfall der Heizkostenkomponente beim Wohngeld und der Wegfall des Zuschlags, mit dem bisher der Übergang vom Arbeitslosengeld (ALG) 1 auf das geringere ALG 2 für einen Zeitraum von 24 Monaten abgemildert wurde.

Darüber hinaus wird der Bundeshaushalt zwar noch durch weitere Maßnahmen wie etwa den Wegfall der Rentenversicherungspflicht für Bezieher von ALG 2 oder den Wegfall der bisher an die GRV geleisteten Erstattungen für einigungsbedingte Leistungen entlastet. Aus Sicht des öffentlichen Gesamthaushalts stellen diese Maßnahmen jedoch nichts weiter als bloße Verschiebebahnhöfe dar, da sich die hierdurch erzielten Einsparungen im Bundeshaushalt bei der GRV als Mindereinnahmen niederschlagen. Für den öffentlichen Gesamthaushalt ist damit nichts gewonnen. Und auch dem Bundeshaushalt ist damit auf Dauer kam geholfen. So werden sich die Mindereinnahmen der GRV spätestens mit Beginn der kommenden Legislaturperiode wieder in einem höheren Bundeszuschuss niederschlagen. Mit anderen Worten: Die notwendige Konsolidierung des Bundeshaushalts wird über den Umweg der GRV lediglich zeitlich in die Zukunft verlagert.

Entsprechend ergibt sich in der Gesamtbetrachtung nur ein geringer Nachhaltigkeitsgewinn infolge des HBeglG und des KernBStG. So reduziert sich die Nachhaltigkeitslücke des öffentlichen Sektors durch diese ersten Schritte des „Zukunftspakets“ lediglich auf 307,8 Prozent des BIP. Im Vergleich zur Nachhaltigkeitslücke bei Amtsantritt (291 Prozent des BIP) ist daher festzuhalten, dass die Nachhaltigkeitsbilanz nach dem ersten Drittel der Legislaturperiode negativ ausfällt. Die bisherigen Konsolidierungsanstrengungen reichten noch nicht einmal aus, um die von Schwarz-Gelb selbst zu verantwortende Belastung der öffentlichen Haushalte durch das WaBeG zu kompensieren. Auch enttäuschen die bisherigen Konsolidierungsschritte insofern, als diese im Wesentlichen durch Steuer- und Abgabenerhöhungen erzielt wurden, während für wirkliche Einsparungen auf der Ausgabenseite offensichtlich der politische Mut fehlte. 
Abbildung 2: Das ,Zukunftspaket“ - alles nur heiße Luft

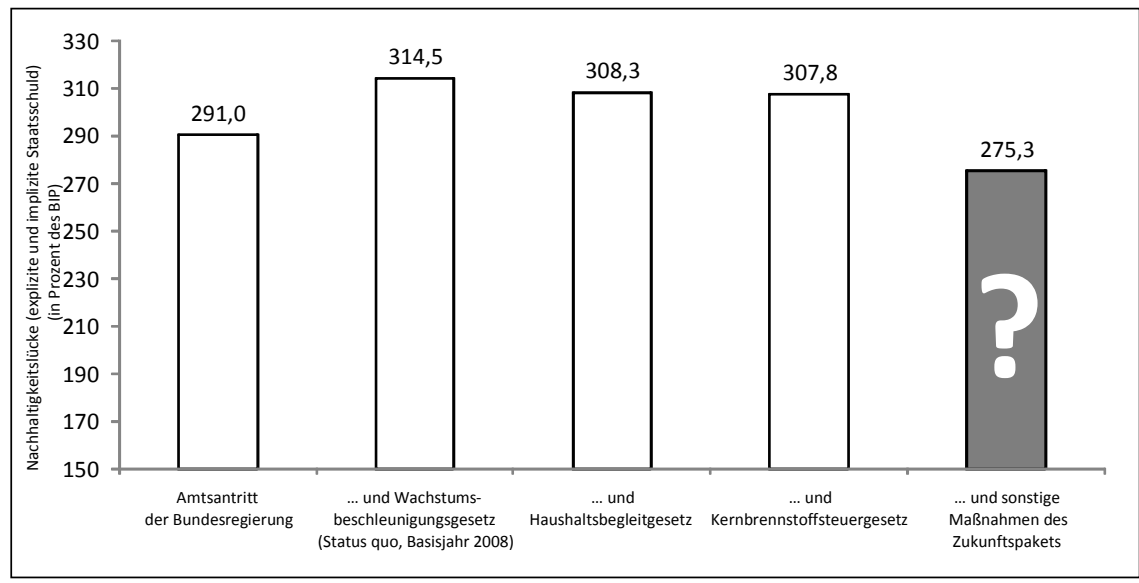

Anmerkung: Basisjahr 2008, g = 1,5\%, $\mathrm{r}=3,0 \%, 12$. koordinierte Bevölkerungsvorausberechnung. Quelle: Eigene Berechnungen.

Als Hoffnungsschimmer bleibt jedoch, dass sich die Nachhaltigkeitslücke der öffentlichen Haushalte bei Umsetzung aller Maßnahmen des „Zukunftspakets“ auf 275,3 Prozent des BIP verringern würde. Damit könnte das „Zukunftspaket“ nicht nur den Anforderungen der neuen Schuldenregel gerecht werden, sondern einen wichtigen Schritt zur langfristigen Haushaltskonsolidierung leisten. Dies hängt jedoch entscheidend davon ab, ob die bisher lediglich als „Luftbuchungen“ geplanten Entlastungen tatsächlich realisiert werden können.

So ist es angesichts der internationalen Widerstände zum Beispiel mehr als fraglich, ob die Finanzmarkttransaktionsteuer, von der sich der Finanzminister zusätzliche Einnahmen in Höhe von 2 Mrd. Euro verspricht, in absehbarer Zeit das Licht der Welt erblicken wird. Und auch die von der Deutschen Bahn an den Bund abzuführende Pflichtdividende in Höhe von 500 Mio. Euro steht auf wackligen Beinen. Denn angesichts des „Winterchaos" “ bei der Deutschen Bahn fordert der Bundesrat, „die von der DB-AG geforderte Dividendenzahlung von 500 Mio. Euro auf das Jahr 2011 zu beschränken“ und diese Mittel stattdessen für Investitionen in die Eisenbahninfrastruktur zu verwenden. ${ }^{10}$

Wesentliche Entlastungen sollen hingegen durch Einsparungen bei den Ausgaben für Arbeitsmarktmaßnahmen und durch die Streitkräftereform realisiert 
werden. Durch die Umwandlung von Pflicht- in Ermessensleistungen ließen sich in der Tat Einsparungen realisieren. Unter Effizienzverbesserungen bei der Arbeitsmarktvermittlung versteht die Bundesregierung hingegen, dass sich durch „optimierte Arbeitsmarktvermittlung und die Rückwirkungen der demografischen Entwicklung [...] mittelfristig eine Belebung des Arbeitsmarktes im SGB II" ergibt, ${ }^{11}$ sodass der Bundeshaushalt ab dem Jahr 2013 entlastet würde. Auf eine Belebung des Arbeitsmarkts infolge der demografischen Entwicklung kann man hoffen. Mit „Sparen“ hat dies allerdings nichts gemein; statt einer seriösen Finanzplanung kann eine solche Politik getrost unter der Rubrik ,finanzpolitisches Wunschdenken" abgelegt werden.

Konkreter erscheinen die Pläne für die Streitkräftereform. Jedoch hat das Verteidigungsministerium bereits deutlich gemacht, dass mit der geplanten Reduzierung der Truppenstärke auf 185.000 Soldaten die anvisierten Einsparungen im Verteidigungsetat nicht zu schaffen sein dürften. ${ }^{12}$ Und auch die Aussetzung der Wehrpflicht wird - zumindest kurzfristig - kaum zu nennenswerten Einsparungen führen.

Davon abgesehen ist festzuhalten, dass das ,Zukunftspaket“ auf dem Papier zwar die Anforderungen der neuen Schuldenregel erfüllt. Der Idee der Schuldenregel wird die Bundesregierung jedoch nicht gerecht. Gemäß den Berechnungen des Sachverständigenrats hätte der Umfang des Konsolidierungsprogramms deutlich größer ausfallen müssen, wenn man bei der Festlegung des Konsolidierungsbedarfs die aktuellen, günstigen Prognosen zur wirtschaftlichen Entwicklung zugrundelegt. ${ }^{13}$ Zwar hat man die gesetzlichen Vorgaben für die Übergangsperiode formal erfüllt. Im Geist der Schuldenbremse hätte man das Konsolidierungsprogramm jedoch an die sich ändernden Rahmenbedingungen anpassen müssen.

\section{Die Gesundheitsreform - den gesundheitspolitischen Stillstand verwalten}

Für die Nachhaltigkeit der öffentlichen Haushalte ist aber selbst dann wenig gewonnen, wenn es dem Bund und den Ländern gelingt, die Vorgaben der Schuldenbremse dauerhaft einzuhalten. Denn so sinnvoll die Schuldenbremse als

11 Bundesministerium für Finanzen: Die Grundpfeiler unserer Zukunft stärken - Acht Punkte für solide Finanzen, neues Wachstum und Beschäftigung und Vorfahrt für Bildung, Berlin, 2010.

12 Vgl. unter Focus Online: Ärger um Sparvorgaben, 15.01.2011.

13 Vgl. Sachverständigenrat, a.a.O., 2010. 
Instrument zur Konsolidierung der Haushalte von Bund und Ländern sein mag, das eigentliche Nachhaltigkeitsproblem der öffentlichen Haushalte ist nach wie vor auf die Konsequenzen der demografischen Entwicklung für die Sozialversicherungen zurückzuführen. Daher war es mehr als zu begrüßen, dass der schwarz-gelbe Koalitionsvertrag für die Gesetzliche Krankenversicherung das Ziel formulierte, das ,[...] bestehende Ausgleichssystem [langfristig] in eine Ordnung mit [...] einkommensunabhängigen Arbeitnehmerbeiträgen“ zu überführen. ${ }^{14}$ Angesichts dieser Zielsetzung erwartete man von der schwarz-gelben Bundesregierung, dass sie den Weg für eine einkommensunabhängige Prämienfinanzierung der GKV freimacht. Diese Erwartung wurde jedoch enttäuscht. Denn im Kern verwaltet die Gesundheitsreform der schwarz-gelben Regierungskoalition bei einigen positiven Ansätzen im Wesentlichen den gesundheitspolitischen Stillstand vergangener Legislaturperioden.

Wie Tabelle 4 zeigt, setzt sich die Reform im weitesten Sinne aus drei Gesetzesvorhaben zusammen: Neben dem GKV-Änderungsgesetz (GKVÄndG) und dem Arzneimittelmarktneuordnungsgesetz (AMNOG) bildet das GKV-Finanzierungsgesetz (GKVFinG) das Kernstück der Gesundheitsreform. Als kurzfristige Maßnahme gegen den anhaltenden Anstieg der Arzneimittelausgaben wurde mit dem GKVÄndG der sogenannte Herstellerabschlag, der der GKV seitens der pharmazeutischen Unternehmen für Arzneimittel ohne Festbetrag zu gewähren ist, befristet bis 2013 von 6 auf 16 Prozent angehoben. Hiervon verspricht sich der Bundesgesundheitsminister Einsparungen bei den Ausgaben für Arzneimittel i.H.v. 1,2 Mrd. Euro jährlich. Das GKVÄndG sieht allerdings nur kurzfristige Einsparungen vor.

Hingegen richtet sich das am 1. Januar 2011 in Kraft getretene AMNOG auf strukturelle und damit dauerhafte Einsparungen bei den Ausgaben für Medikamente. Während für nicht patentgeschützte Arzneimittel mit dem Gesundheitsreformgesetz bereits 1989 sogenannte Festbeträge eingeführt wurden, zielt das AMNOG auf Einsparungen bei den Ausgaben für neue und patentgeschützte Arzneimittel. Konnten die Pharmahersteller für diese Arzneimittel die Preise bisher weitestgehend selbst festlegen, so werden sie zukünftig auf Basis einer Zusatznutzenbewertung ausgehandelt bzw. festgelegt. Auf Basis dieser Bewertung werden neue Medikamente, für die kein Zusatznutzen festzustellen ist, künftig direkt in das Festbetragssystem überführt. Im umgekehrten Fall wird der

14 Vgl. CDU/CSU/FDP: Wachstum, Bildung und Zusammenhalt. Koalitionsvertrag zwischen CDU, CSU und FDP, Berlin, 2009. 
Preis des Medikaments auf Basis der Nutzenbewertung zwischen dem Spitzenverband der Krankenkassen und dem Hersteller ausgehandelt. Insgesamt verspricht sich der Bundesgesundheitsminister durch das AMNOG Einsparungen bei den Ausgaben für Arzneimittel von jährlich 2 Mrd. Euro.

Tabelle 4: Eine Gesundheitsreform in drei Schritten (in Mrd. Euro)

\begin{tabular}{|c|c|c|c|c|c|}
\hline & & 2011 & 2012 & 2013 & 2014 \\
\hline A) & $\begin{array}{l}\text { GKV-Änderungsgesetz } \\
\text { (Anhebung des Herstellerabschlags für Arzneimittel } \\
\text { ohne Festbetrag von } 6 \% \text { auf } 16 \% \text { ) }\end{array}$ & 1,2 & 1,2 & 1,2 & 0,0 \\
\hline B) & $\begin{array}{l}\text { Arzneimittelneuordungsgesetz (AMNOG) } \\
\text { (Nutzenbewertung von neuen Arzneimitteln; Festle- } \\
\text { gung eines Erstattungsbetrags (Rabatts) für die } \\
\text { GKV; Überführung von Medikamenten ohne Zu- } \\
\text { satznutzen in das Festbetragssystem) }\end{array}$ & 2,0 & 2,0 & 2,0 & 2,0 \\
\hline C) & GKV-Finanzierungsgesetz & 7,6 & 7,7 & 6,3 & 6,3 \\
\hline & Anhebung GKV-Beitragssatz von $14,9 \%$ auf $15,5 \%$ & 6,3 & 6,3 & 6,3 & 6,3 \\
\hline & $\begin{array}{l}\text { Einfrieren der Verwaltungskosten auf das Niveau } \\
\text { von } 2010\end{array}$ & 0,3 & 0,3 & \\
\hline & $\begin{array}{l}\text { Begrenzung des Anstiegs der akutstationären Preise } \\
\text { und der Krankenhausbudgets von psychiatrischen } \\
\text { und psychosomatischen Einrichtungen }\end{array}$ & 0,2 & 0,3 & & \\
\hline & Mehrleistungsabschläge bei Krankenhäusern & 0,4 & 0,3 & & \\
\hline & $\begin{array}{l}\text { Begrenzung des Anstiegs der zahnärztlichen Vergü- } \\
\text { tung für Zahnbehandlung }\end{array}$ & 0,0 & 0,0 & & \\
\hline & $\begin{array}{l}\text { Begrenzung der Vergütungen im Bereich der haus- } \\
\text { arztzentrierten Versorgung }\end{array}$ & 0,5 & 0,5 & & \\
\hline \multicolumn{2}{|r|}{$\begin{array}{l}\text { Gesamte Entlastung der Gesetzlichen Krankenversi- } \\
\text { cherung }\end{array}$} & 10.8 & 10,9 & 9,5 & 8,3 \\
\hline \multicolumn{2}{|r|}{ Mehrbelastung der Gebietskörperschaften } & -0.8 & $-\mathbf{0 , 8}$ & $-0,8$ & $-0,8$ \\
\hline & $\begin{array}{l}\text { Mehrausgaben der Gebietskörperschaften in ihrer } \\
\text { Funktion als Arbeitgeber }\end{array}$ & $-0,2$ & $-0,2$ & $-0,2$ & -0.2 \\
\hline & $\begin{array}{l}\text { Mindereinnahmen bei der Einkommensteuer infolge } \\
\text { höherer Vorsorgeaufwendungen }\end{array}$ & $-0,6$ & $-0,6$ & $-0,6$ & -0.6 \\
\hline \multicolumn{2}{|r|}{ Gesamte Entlastung des öffentlichen Gesamthaushalts } & 10.0 & 10,1 & 8,7 & 7,5 \\
\hline
\end{tabular}

Quelle: Ausschuss-Drucks. 17(14)0034, BR-Drucks. 581/10, BT-Drucks. 17/ $297,17 / 2413$. 
Das Kernstück der Gesundheitsreform bildet das GKVFinG, mit dem im Wesentlichen zwei Ziele verfolgt werden. Zum einen wollte der Gesetzgeber sicherstellen, dass das für das Jahr 2011 zu erwartende Defizit der GKV gedeckt ist. Neben kurzfristigen Einsparungen wurde hierzu der allgemeine Beitragssatz zur GKV zum 1. Januar 2011 von 14,9 auf 15,5 Prozent angehoben. Als eigentliche Reformmaßnahme wurde mit dem GKVFinG zum anderen das bestehende System der Zusatzbeiträge weiterentwickelt. Hierzu schaffte man einerseits die sogenannte Deckungsquote ab, mit der die Höhe der Zusatzbeiträge bisher auf maximal 5 Prozent der Ausgaben gedeckelt wurde. Als Konsequenz kann der Ausgabenanstieg im Gesundheitswesen zukünftig in vollem Umfang durch Zusatzbeiträge finanziert werden. Andererseits wird der bisher auf Kassenebene angesiedelte Sozialausgleich zukünftig aus Steuermitteln finanziert. ${ }^{15}$ Die hiermit eingeschlagene Richtung ist positiv zu bewerten, da sie den Wettbewerb zwischen den Krankenkassen befördert und die beschäftigungsfeindliche Anknüpfung der Beiträge an die Lohneinkommen in der Zukunft nicht noch weiter verschärft.

\section{Abbildung 3: Die Gesundheitsreform}

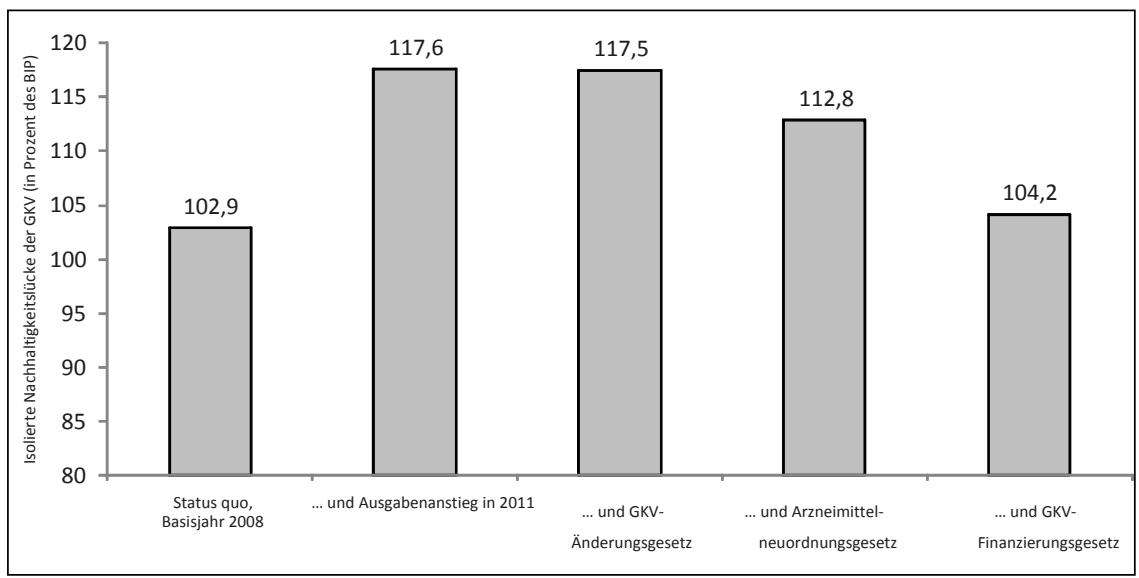

Anmerkung: Basisjahr 2008, g = 1,5\%, r = 3,0 \%, 12. koordinierte Bevölkerungsvorausberechnung. Quelle: Eigene Berechnungen.

15 Allerdings erfolgt ein Sozialausgleich erst dann, wenn der durchschnittliche Zusatzbeitrag zwei Prozent des individuellen beitragspflichtigen Einkommens übersteigt. Hingegen erfolgte der Sozialausgleich nach alter Regelung bereits dann, wenn der tatsächliche Zusatzbeitrag ein Prozent des individuellen beitragspflichtigen Einkommens überschritt, wobei die Mittel zur Finanzierung des Sozialausgleichs durch die einzelne Krankenkasse aufgebracht werden mussten. 
Insgesamt ist mit der Gesundheitsreform für die Nachhaltigkeit der GKV jedoch wenig gewonnen. Wie Abbildung 3 zeigt, bietet diese lediglich eine kurzfristige Vermeidung weiterer Defizite der GKV. Lässt man die Wirkungen der Gesundheitsreform zunächst außer Acht, so hätte sich die Nachhaltigkeit der GKV allein infolge des für das Jahr 2011 prognostizierten Anstiegs der Gesundheitsausgaben auf 117,6 Prozent des BIP verschlechtert. Diesem ausgabeninduzierten Nachhaltigkeitsverlust wirken die drei Reformgesetze der Bundesregierung zwar entgegen, doch verbleibt eine Nachhaltigkeitslücke von 104,2 Prozent des BIP; mit der Gesundheitsreform wurde für die Nachhaltigkeit der GKV mithin wenig gewonnen.

In der Gesamtbetrachtung ist daher festzuhalten, dass die Gesundheitsreform der schwarz-gelben Koalition sich weitestgehend in die lange Tradition gesundheitspolitischer Fehlschläge einreiht. Mittels Beitragssatzerhöhungen und kurzfristiger Kostendämpfungsmaßnahmen werden im Wesentlichen wieder nur die Symptome der anhaltenden Misere im deutschen Gesundheitswesen kuriert. Positiv zu bewerten ist jedoch, dass die GKV-Finanzierung einen neuen Weg einschlägt: Statt über steigende Beitragssätze sollen die Kostensteigerungen künftig allein über Zusatzbeiträge finanziert werden. Dies vermeidet zumindest eine weitere Belastung des Arbeitsmarktes und forciert den Wettbewerb zwischen den Kassen. Im Ansatz sinnvoll ist auch die Weiterentwicklung des Sozialausgleichs, obwohl ein ,gesamtgesellschaftlicher Ausgleich“ auch damit nicht gelingt. Denn wie im bisherigen System werden für die Ausgleichszahlungen statt des tatsächlichen Einkommens lediglich die beitragspflichtigen Einnahmen zugrundegelegt. $\mathrm{Zu}$ begrüßen ist schließlich das AMNOG, da es zumindest das Potential für eine dauerhafte (Teil-)Lösung der Begrenzung des Ausgabenanstiegs im Arzneimittelbereich beinhaltet.

\section{Fazit}

Nach dem wirtschaftlich erfolgreichsten Jahr seit der Wiedervereinigung wird der krisenbedingte Einbruch der Wirtschaftsleistung angesichts des anhaltenden konjunkturellen Aufschwungs binnen Jahresfrist kompensiert sein. Entgegen allen Prognosen hat sich die deutsche Volkswirtschaft damit unerwartet schnell von den Folgen der Wirtschafts- und Finanzkrise erholt. Und obwohl sich auch die für das Jahr 2010 veranschlagte Rekordneuverschuldung von $86 \mathrm{Mrd}$. Euro infolge sprudelnder Steuereinnahmen auf $50 \mathrm{Mrd}$. Euro verringert hat, fällt die fiskalische Bilanz von Schwarz-Gelb bisher ernüchternd aus. Mit dem „Wachstumsbeschleunigungsgesetz“, dem „Zukunftspaket“ und der Gesundheitsreform 
wurden zwar wesentliche wirtschaftspolitische Ziele des Koalitionsvertrages angegangen. Insgesamt konnte die Bundesregierung die in sie gesetzten Erwartungen jedoch nicht erfüllen, vielmehr enttäuschte sie durch ihre Mutlosigkeit.

Zur Bekämpfung der Folgen der Wirtschafts- und Finanzkrise wollte die Koalition mit dem „Wachstumsbeschleunigungsgesetz“ neue Impulse für einen dynamischen Aufschwung setzen. Die Realität sieht jedoch anders aus. Bei einem vernachlässigbaren Einfluss auf die Wirtschaftsleistung hatten die beschlossenen Steuererleichterungen lediglich eine Zunahme des strukturellen Defizits zur Folge und bescherten den öffentlichen Haushalten eine zusätzliche Zukunftshypothek. Die Antwort auf die Frage der Gegenfinanzierung dieser Steuererleichterungen blieb die schwarz-gelbe Bundesregierung bis zum Sommer 2010 schuldig. So bedurfte es erst fallender Umfragewerte, bis sich die Koalition mit dem „Zukunftspaket“ auf eine Konsolidierungsstrategie verständigte, die zumindest auf dem Papier den Anforderungen der Schuldenbremse genügt. Allerdings muss auch diese Maßnahme als mutlos gelten. Zwar wurden mit dem Haushaltsbegleitgesetz und dem Kernbrennstoffsteuergesetz bereits erste konkrete Maßnahmen umgesetzt, doch bleibt angesichts des inzwischen beschlossenen Atomausstiegs fraglich, ob und wie das eingeplante Aufkommen der Kernbrennstoffsteuer tatsächlich realisiert werden kann.

Auch im günstigsten Fall wären diese Maßnahmen noch nicht ausreichend, um die von Schwarz-Gelb selbst zu verantwortende Belastung der öffentlichen Haushalte zu kompensieren. Davon abgesehen bietet das „Zukunftspaket“ lediglich Absichtserklärungen und Hoffnungswerte. Einen nennenswerten Beitrag zur Haushaltskonsolidierung wird man ihm daher erst dann zusprechen können, wenn es der Bundesregierung gelingt, die bisher lediglich als „Luftbuchungen“ auf dem Papier vorhandenen Konsolidierungsmaßnahmen in die Tat umzusetzen. Allerdings wird auch dies kaum ausreichen, um die Haushalte von Bund, Ländern und Gemeinden dauerhaft zu konsolidieren. Hierzu sind weitere Maßnahmen unumgänglich, wie etwa die wirkungsgleiche Übertragung der bereits in der gesetzlichen Rentenversicherung beschlossenen Reformschritte auf die pensionierte Beamtenschaft. Allein die damit verbundene Gleichbehandlung würde das Nachhaltigkeitsproblem der Gebietskörperschaften dauerhaft um etwa ein Drittel senken.

Als Unzureichend erwies sich schließlich auch die Gesundheitsreform der Bundesregierung. Mit der Weiterentwicklung der Zusatzbeiträge wird die Finanzierung der GKV zwar graduell durch einkommensunabhängige Prämienelemente ergänzt, womit der Arbeitsmarkt vom Anstieg der Gesundheitsausgaben entkop- 
pelt und der Wettbewerb zwischen den Krankenkassen gestärkt wird. Allein damit ist für die Nachhaltigkeit der GKV jedoch wenig gewonnen. Denn statt über steigende Beitragssätze wird der Anstieg der Gesundheitsausgaben nur über steigende Zusatzbeiträge finanziert. Und da die demografische Herausforderung im Gesundheitswesen ohne eine Reform der Ausgabenseite nicht zu bewältigen ist, hätte man mit etwas Mut zumindest einen ersten Schritt für eine wirklich nachhaltige und damit generationengerechte Reform leisten können. Hierzu wäre es jedoch erforderlich, den einkommensabhängigen Beitragssatz zumindest teilweise durch eine einkommensunabhängige Gesundheitsprämie abzulösen. Stattdessen wird der demografisch bedingte Anstieg der Gesundheitsausgaben wie generell nahezu aller Ausgaben - auf die Schultern der nachkommenden Generationen abgewälzt. Die schwarz-gelbe Bundesregierung scheint sich der Problematik dieser Erkenntnis noch nicht bewusst. 